\title{
Synthesis, thermal, dielectric, and microwave reflection loss properties of nickel oxide filler with natural fiber-reinforced polymer composite.
}

\begin{abstract}
Fabrication of hybrid composite of nickel oxide $(\mathrm{NiO})$ combined with oil palm empty fruit bunch (OPEFB) reinforced with polycaprolactone (PCL) has been done by using thermal Haake blending machine, which ensured mixture homogeneity. All hybrid composites' characterizations were carried out using X-ray diffraction (XRD), Fourier transform infrared spectrometry, differential thermogravimetry, thermogravimetric analysis, and scanning electron microscopy. The results showed that the XRD profile patterns of the composites clearly changed as the filler loading amount was increased. Fourier transform infrared spectra illustrated a slight change in the frequencies and positions of the peaks after adding $\mathrm{NiO}$, indicating that some interactions occurred between $\mathrm{C}=\mathrm{O}$ and $\mathrm{O}-\mathrm{H}$ or among the fiber, NiO, and PCL. The microwave electromagnetic properties, such as reflection loss $(\mathrm{dB})$, relative complex permittivity $(\varepsilon \mathrm{r}=$ (Formula presented.) $-\mathrm{j}$ (Formula presented.)), and permeability ((Formula presented.) $-\mathrm{j}$ (Formula presented.)) were calculated at various microwave frequencies in the X-band (8-12 $\mathrm{GHz}$ ) range. It was observed that the thermal stability, magnetic, and dielectric properties of NiO:OPEFB:PCL composites were modified significantly with $\mathrm{NiO}$ addition. This enables the new hybrid composites to be used as engineering materials in the microwave applications.
\end{abstract}

Keyword : Microwave; Polycaprolactone; Polymer composites; Reflection loss; Relative complex. 